\title{
Structure-activity relationships of the plasminogen modulator SMTP with respect to the inhibition of soluble epoxide hydrolase
}

\begin{abstract}
Naoki Matsumoto $^{1}$, Eriko Suzuki ${ }^{1}$, Kota Tsujihara ${ }^{1}$, Yuuichi Nishimura ${ }^{1}$ and Keiji Hasumi ${ }^{1,2}$
A family of fungal metabolites, SMTP, is a small-molecule plasminogen modulator that enhances plasminogen activation, leading to thrombolysis. We recently demonstrated that SMTP-7 effectively treats ischemic stroke due to its thrombolytic activity as well as anti-inflammatory action, which is attributable to soluble epoxide hydrolase (sEH) inhibition. In this paper, we studied detailed structure-activity relationships of plasminogen modulation and SEH inhibition using 25 SMTP congeners including six newly synthesized ones. The results clearly demonstrate that the structure of the $\mathrm{N}$-linked side chain of SMTP congeners markedly affect their activities toward plasminogen modulation and inhibitions of the two activities of $\mathrm{SEH}$ (C-terminal epoxide hydrolase and $\mathrm{N}$-terminal phosphatase). A slight change in the $\mathbf{N}$-linked side chain results in affording selectivity of SMTP congeners. Many congeners, which lacked plasminogen modulation activity, differently inhibited the two sEH activities depending on the structures of the $\mathbf{N}$-linked side chain. Some congeners were active in plasminogen modulation and inhibition of both activities of SEH. These results help comprehensive understanding of ideal design of a drug useful for ischemic diseases that are associated with inflammation, such as stroke.
\end{abstract}

The Journal of Antibiotics (2015) 68, 685-690; doi:10.1038/ja.2015.58; published online 13 May 2015

\section{INTRODUCTION}

SMTPs (named after Stachybotrys microspora triprenyl phenols) are a family of novel small molecules produced by the fungus S. microspora. ${ }^{1-15}$ SMTP-7, one of the SMTP family compounds with profound biological activities, enhances proteolytic activation of plasminogen, a circulating zymogen of plasmin, which is the major protease responsible for blood clot dissolution. ${ }^{1}$ This activity of SMTP-7 is attributable to the modulation of plasminogen conformation: although plasminogen is resistant to proteolytic activation due to spiral, closed conformation, SMTP-7 relaxes its conformation to an activation-prone conformation. ${ }^{1,6,14,15}$ SMTP-7 thus promotes physiological plasmin formation, clot clearance in vivo ${ }^{14,16}$ and thereby effectively treats thrombotic and embolic strokes in animal models in rodents and primates. ${ }^{17-21}$ Notably, the action of SMTP-7 is accompanied by reduced hemorrhagic transformation ${ }^{17,20,21}$ and a wide therapeutic time window. ${ }^{17,18}$ The excellent efficacy of SMTP-7 is partly explained by its anti-inflammatory and antioxidative activities. . $^{16,17,19,21-23}$ On the basis of these properties, we are developing SMTP congeners as a drug for treatment of stroke, a major cause of death and disability worldwide. ${ }^{24,25}$ One of the SMTP congeners is under a phase I clinical trial, which is led by TMS, Japan.

Our recent investigations have demonstrated that the antiinflammatory action of SMTP is independent of its thrombolytic activity, and that soluble epoxide hydrolase $(\mathrm{sEH})$ is involved in the anti-inflammatory action. ${ }^{26} \mathrm{sEH}$ is a bifunctional enzyme with an epoxide hydrolase activity at the C-terminal domain (Cterm-EH) and a lipid phosphate phosphatase activity at the $\mathrm{N}$-terminal domain (Nterm-phos). ${ }^{27}$ The Cterm-EH catalyzes the hydrolysis of epoxy-fatty acids such as epoxyeicosatrienoic acids, which are potent endogenous signaling molecules implicated in anti-inflammation, vascular dilation, endothelial cell hyperpolarization, angiogenesis, neuroprotection and analgesia (antihyperalgesia). ${ }^{28-32}$ The Nterm-phos hydrolyzes lipid phosphates, such as lysophosphatidic acid, and intermediates of the cholesterol biosynthesis. ${ }^{33-35}$

The SMTP molecule consists of a tricyclic $\gamma$-lactam moiety, an isoprene side chain and an $N$-linked side chain, which only differs among congeners. ${ }^{1-3,5-8,10-14}$ Some SMTP congeners inhibited both of the two activities of sEH. ${ }^{26}$ The mode of inhibition by SMTP-0, the simplest SMTP congener without plasminogen modulation activity, ${ }^{8}$ is competitive for Cterm-EH and pseudo-noncompetitive for Ntermphos. ${ }^{26}$ A preliminary investigation has revealed that the selectivity of the inhibition of the two sEH activities greatly changed depending on the $N$-linked side-chain structure, demonstrating that SMTP binds to two distinct sites in $\mathrm{sEH} .{ }^{26}$ In conjunction with that the $N$-linked side chain is a crucial determinant for plasminogen modulation activity of SMTP, ${ }^{10-12,14}$ it is possible that changing the $N$-linked side-chain structure leads to the generation of an SMTP congener specific or universal for each of the three targets, plasminogen, Cterm-EH and Nterm-phos. In this study, we tested a variety of SMTP congeners (25 including 6 new congeners) for their activities in inhibitions of 
sEH Cterm-EH and Nterm-phos in comparison with plasminogen modulation to understand structure-activity relationships that span the three targets.

\section{MATERIALS AND METHODS}

\section{Materials}

Human native plasminogen (Glu1-plasminogen) was isolated on LysineSepharose affinity chromatography. Two-chain urokinase-type plasminogen activator was purchased from JCR Pharmaceuticals (Kobe, Japan). SMTP congeners as well as pre-SMTP were prepared as described previously. ${ }^{5-8,10-14}$ Solutions of SMTPs in dimethyl sulfoxide were used for assays for plasminogen activation, as well as Cterm-EH and Nterm-phos of sEH (the concentration of dimethyl sulfoxide was kept at less than $1 \%, \mathrm{v} / \mathrm{v}$ ).

\section{Production of new SMTP congeners}

SMTP-52, - -57, -58, -60, -61 and -62 were produced by a single-step reaction between pre-SMTP ${ }^{13}$ and either of the following amines: asparagine, 2-aminopropane, cyclohexylamine, cyclohexylmethylamine, 1-aminocyclohexanecarboxylic acid and trans-4-aminocyclohexanol, respectively. PreSMTP $(10 \mathrm{mg}$ ) dissolved in $1.25 \mathrm{ml}$ of methanol was added to $4 \mathrm{ml}$ of water$\mathrm{MeOH}$-acetic acid (75:12.5:12.5) containing excess amount of either of the amines described above. The reaction, which allowed the incorporation of the added amine as the $N$-linked side chain, ${ }^{13}$ was performed at $50^{\circ} \mathrm{C}$ for $2 \mathrm{~h}$. The resulting SMTP congeners were purified by reverse-phase HPLC with a gradient of $\mathrm{MeOH}$ in $0.1 \%(\mathrm{w} / \mathrm{v})$ formic acid.

\section{Assay for plasminogen activation}

The activation of plasminogen was assayed by measuring initial velocity for tcu-PA-catalyzed plasmin generation using the chromogenic substrate H-Val-Leu-Lys-p-nitroanilide (VLK-pNA; Bachem, Bubendorf, Switzerland). A reaction mixture consisting of $50 \mathrm{~nm}$ plasminogen, $50 \mathrm{U} \mathrm{ml}^{-1}$ two-chain urokinase-type plasminogen activator and $0.1 \mathrm{~mm}$ VLK-pNA in $50 \mu \mathrm{l}$ of buffer (50 mm Tris-HCl, $100 \mathrm{~mm} \mathrm{NaCl}$ and $0.01 \%$ Tween $80, \mathrm{pH} 7.4$ ) was incubated in the presence or absence of SMTP congeners at $37^{\circ} \mathrm{C}$. The hydrolysis of VLK-pNA (absorbance at $405 \mathrm{~nm}$ ) was kinetically monitored for up to $60 \mathrm{~min}$. From the slope of the plots of $\mathrm{A}_{405}$ versus $t^{2}$, the initial velocity of plasmin generation was calculated. The concentration of SMTP that caused a 10 -fold enhancement $\left(\mathrm{EC}_{10}\right)$ and the maximum level of the enhancement $\left(E_{\max }\right)$ were estimated from the plots of fold of enhancement of plasminogen activation versus SMTP concentration. From these data, the comprehensive potency of plasminogen modulation $\left(E_{\max } / \mathrm{EC}_{10}\right)$ was calculated. ${ }^{10-12}$

\section{Assay for $\mathrm{sEH}$}

The Cterm-EH activity was assayed using (3-phenyl-oxiranyl)-acetic acid cyano-(6-methoxy-naphthalen-2-yl)-methyl ester (PHOME) as a substrate. Purified mouse liver $\mathrm{sEH}^{26}$ (60 ng) was preincubated for $10 \mathrm{~min}$ in $80 \mu \mathrm{l}$ of $25 \mathrm{~mm}$ Bis-Tris-HCl, $\mathrm{pH} 7.0$, containing $0.1 \mathrm{mM} \mathrm{MgCl}_{2}$ and $0.1 \mathrm{mg} \mathrm{ml}^{-1}$ bovine serum albumin (buffer A) with or without a compound to be tested. The composition of buffer A was based on the method by Tran et al., ${ }^{33}$ unless $\mathrm{MgCl}_{2}$ was added to unify the buffer composition with that for the Nterm-phos determination, in which $\mathrm{MgCl}_{2}$ is essential. After adding $20 \mu \mathrm{l}$ of the substrate, fluorescence (excitation, $355 \mathrm{~nm}$; emission, $460 \mathrm{~nm}$ ) of the reaction product was measured kinetically at $30^{\circ} \mathrm{C}$. The final concentrations of sEH and PHOME were $4.7 \mathrm{~nm}$ and $12.5 \mu \mathrm{M}$, respectively. The Nterm-phos activity was assayed using AttoPhos as a substrate. Mouse sEH (30 ng) was preincubated for $10 \mathrm{~min}$ in $80 \mu \mathrm{l}$ of buffer A with or without a compound to be tested. After adding $20 \mu \mathrm{l}$ of AttoPhos, fluorescence (excitation, $450 \mathrm{~nm}$; emission, $545 \mathrm{~nm}$ ) of the reaction product was measured kinetically at $30^{\circ} \mathrm{C}$. The final concentrations of sEH and AttoPhos were $2.3 \mathrm{~nm}$ and $5 \mu \mathrm{M}$, respectively.

\section{General procedures}

UV spectrum was measured in $\mathrm{MeOH}$ on a model 320 spectrometer (Hitachi, Tokyo, Japan) and IR spectrum on a JIR-WINSPEC (JEOL, Tokyo, Japan) with a $\mathrm{NaCl}$ plate (neat). Mass spectrum was taken on a model JMS-T100LP (JEOL) using an ESI method. NMR spectra were measured in methanol- $d_{4}$ (for SMTP-52) or acetone- $d_{6}$ (for SMTP-57, -58, -60, -61, and -62) on a JNMAlpha-400 (JEOL) (for SMTP-57) or a JNM-Alpha-600 (JEOL) (for SMTP-52, $-58,-60,-61$, and -62$)$.

\section{RESULTS AND DISCUSSION}

Plasminogen modulation activities of the newly synthesized SMTP congeners

To expand the diversity of SMTP library for structure-activity relationship studies, we prepared six new congeners (SMTP-52, -57, $-58,-60,-61$ and -62$)$ using the chemical method employing a singlestep reaction between pre-SMTP and an amine compound to be introduced as the $\mathrm{N}$-linked side chain. ${ }^{13}$ The physicochemical properties and NMR spectral data (Table 1; Supplementary Figures 1a-6b) for the new congeners were consistent with those for the predicted structures.

The six new congeners as well as two uncharacterized congeners (SMTP-54 and -55$)^{13}$ were tested for plasminogen modulation. The results indicated that only SMTP-61 was active (Figure 1). Its concentrations affording 10-fold enhancement $\left(\mathrm{EC}_{10}\right)$ and maximum enhancement $\left(E_{\max }\right)$ were $110 \mu \mathrm{M}$ and 16-fold, respectively. The potency of plasminogen modulation, expressed as the ratio of $E_{\max }$ over $\mathrm{EC}_{10}$, was 0.15 -fold per $\mu \mathrm{M}$, which was $<1 / 10$ of that of SMTP-7 (1.57-fold per $\left.\mu \mathrm{M}^{-1}\right){ }^{11,12}$ These results are consistent with the previous findings that an $\mathrm{N}$-linked side chain with both an aromatic and a negatively ionizable groups is essential for potent plasminogen modulation activity. ${ }^{10-12}$

\section{Structure-activity relationships with respect to plasminogen modulation and Cterm-EH inhibition}

Although seven of eight currently evaluated SMTP congeners were inactive in plasminogen modulation, some of them were effective in inhibiting Cterm-EH of sEH (Figure 2a, left panel). SMTP-52, -61 and -62 were inhibitory to Cterm-EH ( $\mathrm{IC}_{50} 36$ and $48 \mu \mathrm{M}$, respectively), whereas SMTP-58 and -60, which lack the side-chain carboxyl or hydroxyl group of SMTP-58, were essentially inactive $\left(\mathrm{IC}_{50}>100 \mu \mathrm{M}\right)$. Similarly, SMTP-16, $-33,-34,-38$ and -57 , which do not have a negatively ionizable side chain, were much less active (Figure $2 \mathrm{a}$, left panel). The only exception was SMTP-0, which lacks the $N$-linked side chain. SMTP-0 $\left(\mathrm{IC}_{50}\right.$ for Cterm-EH=6 $\left.=6 \mathrm{M}\right)$ was one of the potent congeners tested so far. ${ }^{26}$ Other congeners with a negatively ionizable group at the $\mathrm{N}$-linked side chain were generally inhibitory $\left(\mathrm{IC}_{50}<50 \mu \mathrm{M}\right)$ to Cterm-EH (SMTP-4, -5, -6, -7, -13, -14, -18, 19, -23, $-24,-25,-26,-27,-28,-42,-43,-44,-44 \mathrm{D},-52,-54$ and -55$)$. Of the congeners with relatively high plasminogen modulation activity with $E_{\max } / \mathrm{EC}_{10}>0.4$-fold per $\mu$ M (SMTP-7, -14, -19, -21, -23, -25, -28, -42, -43 and $-43 \mathrm{D}$ ), only SMTP-21 was weak in Cterm-EH inhibition $\left(\mathrm{IC}_{50}>60 \mu \mathrm{M}\right)$ (Figure 2a, left panel).

Consistent with the previous study, ${ }^{26}$ these results clearly demonstrate that plasminogen modulation and Cterm-EH inhibition require a distinct structural feature of the $\mathrm{N}$-linked side chain. Although an $\mathrm{N}$-linked side chain with an aromatic and a negatively ionizable groups is essential for plasminogen modulation, only the core unit of SMTP (a tricyclic $\gamma$-lactam with a geranylmethyl group) is sufficient for the inhibition of Cterm-EH. Nevertheless, certain structural features of the $\mathrm{N}$-linked side chain, such as the presence or absence of the carboxyl or hydroxyl group as well as its position, can modulate the potency of Cterm-EH (Figure 2a, left panel). 


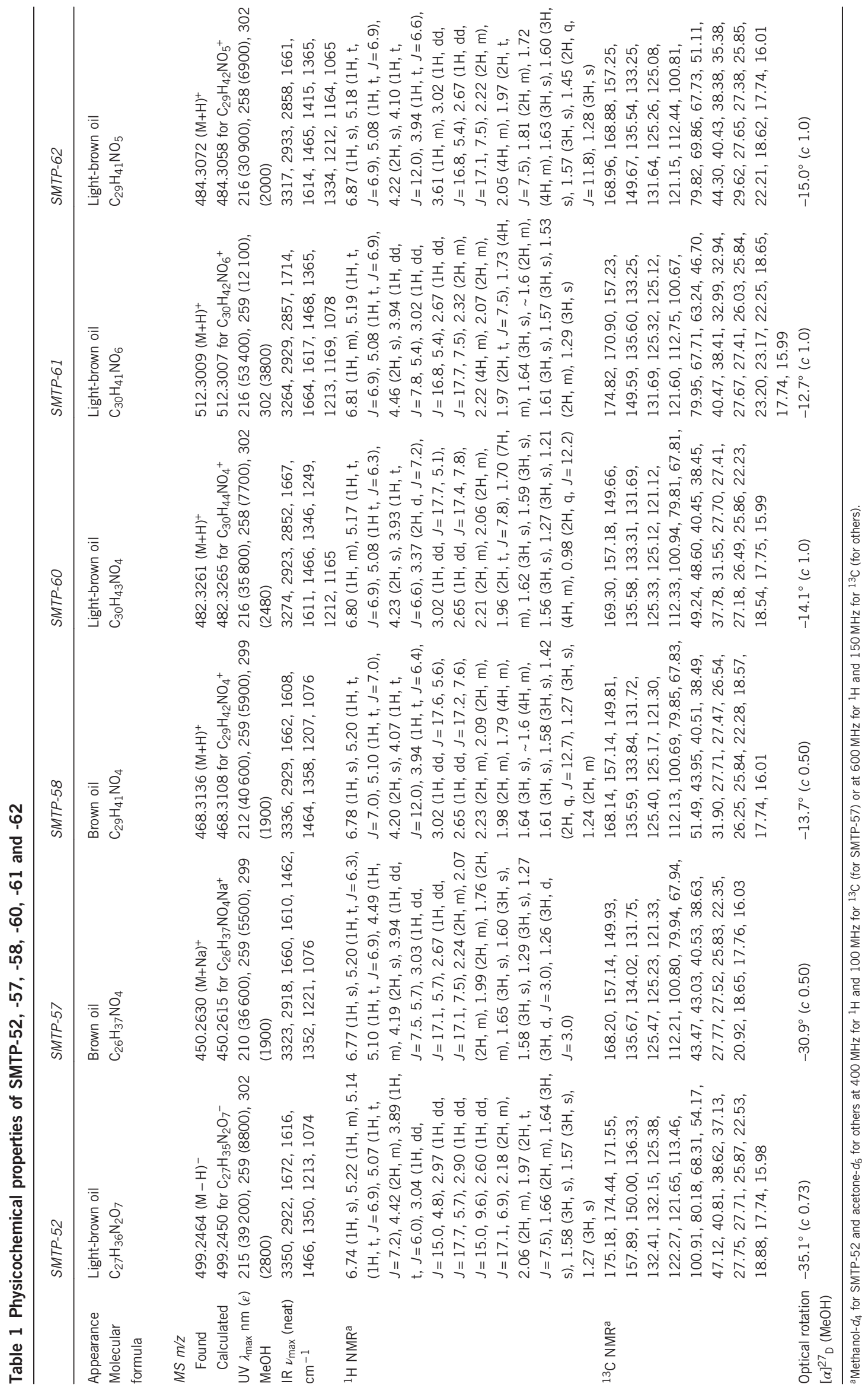


a

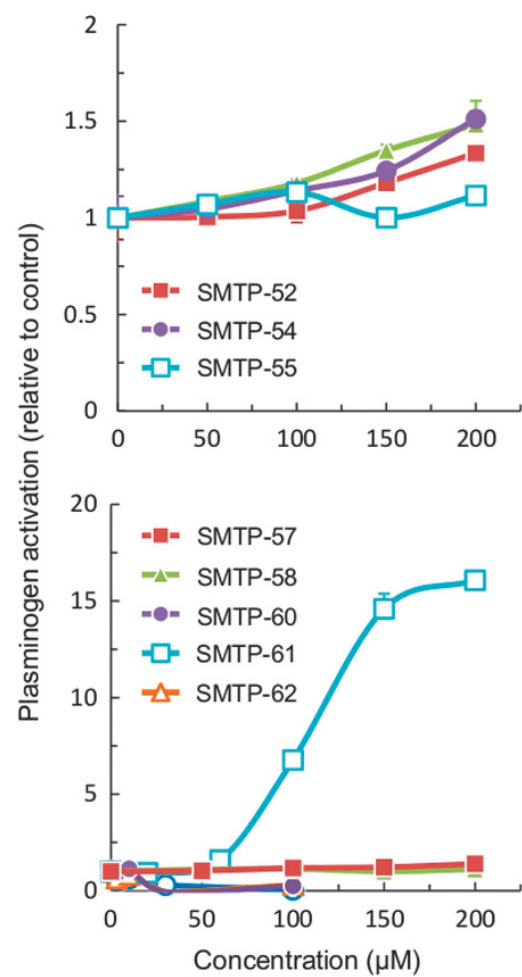

b

Compound

Figure 1 Structures and plasminogen modulation activities of the new SMTP congeners. (a) Plasminogen modulation activity was assessed as the activation of plasminogen. This was assayed in the presence of the indicated concentrations of each SMTP congener. Each value represents the mean \pm s.d. from triplicate determinations. Relative activities (control $=1$ ) are shown. (b) Structures of the new congeners and the summary of the results in a. $\mathrm{EC}_{10}$, concentration ( $\mu$ ) of SMTP that causes 10-fold enhancement of plasminogen activation; $E_{\max }$, maximum level of enhancement (fold increase in plasminogen activation compared with control). $E_{\max }$ and the reciprocal of $\mathrm{EC}_{10}$ are independent indexes that represent the potency of the compound tested. The ratio $E_{\max } / E_{10}$ represents comprehensive potency. $\mathrm{NA}$, not available (as enhancement did not reach 10-fold at concentrations tested).

\section{Structure-activity relationships with respect to plasminogen modulation and Nterm-phos inhibition}

Many of the congeners with little or no plasminogen modulation activity $\left(E_{\max } / \mathrm{EC}_{10}<0.1\right.$-fold $\left.\mu \mathrm{M}^{-1}\right)$ were inhibitory to Nterm-phos $\left(\mathrm{IC}_{50}<50 \mu \mathrm{M}\right)($ SMTP-0, $-4,-5,-11,-12,-18,-26,-44,-57,-58$ and -60$)$ as did all the congeners with relatively potent plasminogen modulation activity $\left(E_{\max } / \mathrm{EC}_{10}>0.4\right.$-fold $\mu \mathrm{M}^{-1}$ ) (SMTP-7, -14, -19, $-21,-23,-25,-28,-42,-43$ and $-43 \mathrm{D}$ ) (Figure $2 \mathrm{a}$, right panel). The congeners with little or no inhibitory activity toward Nterm-phos $\left(\mathrm{IC}_{50}>50 \mu \mathrm{M}\right)$ were SMTP-34, $-38,-52$ and -62 . All of these were inactive in plasminogen modulation. In summary, the structural requirement for plasminogen modulation and Nterm-phos inhibition is apparently different, and there are several congeners that potently inhibit Nterm-phos without plasminogen modulation activity (SMTP-4 and -5$)$. In general, congeners with high plasminogen modulation activities $\left(E_{\max } / \mathrm{EC}_{10}>0.9\right.$-fold per $\left.\mu \mathrm{M}\right)(\mathrm{SMTP}-7,-19,-25$ and -43$)$ are effective inhibitors of Nterm-phos, whereas the potencies of SMTP-14 and -21 were low (Figure 2a, right panel).

\section{Structure-activity relationships with respect to Cterm-EH and Nterm-phos inhibition in combination with plasminogen modulation}

The above-mentioned results are summarized in Figure $2 \mathrm{~b}$ for comprehensive analysis. SMTP-7, -19 and -25 are active among the three activities, plasminogen modulation and inhibitions of Cterm-EH and Nterm-phos. SMTP-0 and -26 are inhibitory to both Cterm-EH and Nterm-phos of sEH but inactive in plasminogen modulation. SMTP-5D and -43D have significant activities in plasminogen modulation and Nterm-phos inhibition, but have very weak activities in Cterm-EH inhibition. SMTP-54 and -55 are inhibitory to Cterm-EH but very weak in Nterm-phos inhibition and plasminogen modulation. SMTP-58 and -60 are inactive in plasminogen modulation and Cterm-EH inhibition but inhibitory to Nterm-phos. SMTP-16, -33 and -38 are totally inactive in the three parameters.

\section{CONCLUSION}

The present study clearly demonstrates that the structure of the $\mathrm{N}$-linked side chain of SMTP congeners markedly affect their activities toward plasminogen modulation and inhibitions of Cterm-EH and Nterm-phos of sEH. Although the core unit of SMTP is sufficient to inhibit Cterm-EH and Nterm-phos, as exemplified by SMTP-0, a slight change in the $N$-linked side chain can afford a selective congener. sEH-null mice are resistant to development of acute inflammation, sepsis, ischemia/reperfusion injury in the heart and brain, inflammatory bowel disease, diabetes and pulmonary hypertension. ${ }^{36}$ Thus, the simultaneous inhibition of Cterm-EH and Nterm-phos of sEH is an ideal means of pharmacological intervention of the above-mentioned disease conditions. In addition, our previous results have demonstrated that the combination of thrombolysis (attributable to plasminogen modulation) and anti-inflammation (mediated by sEH inhibition) by SMTP-7 contributes to excellent 

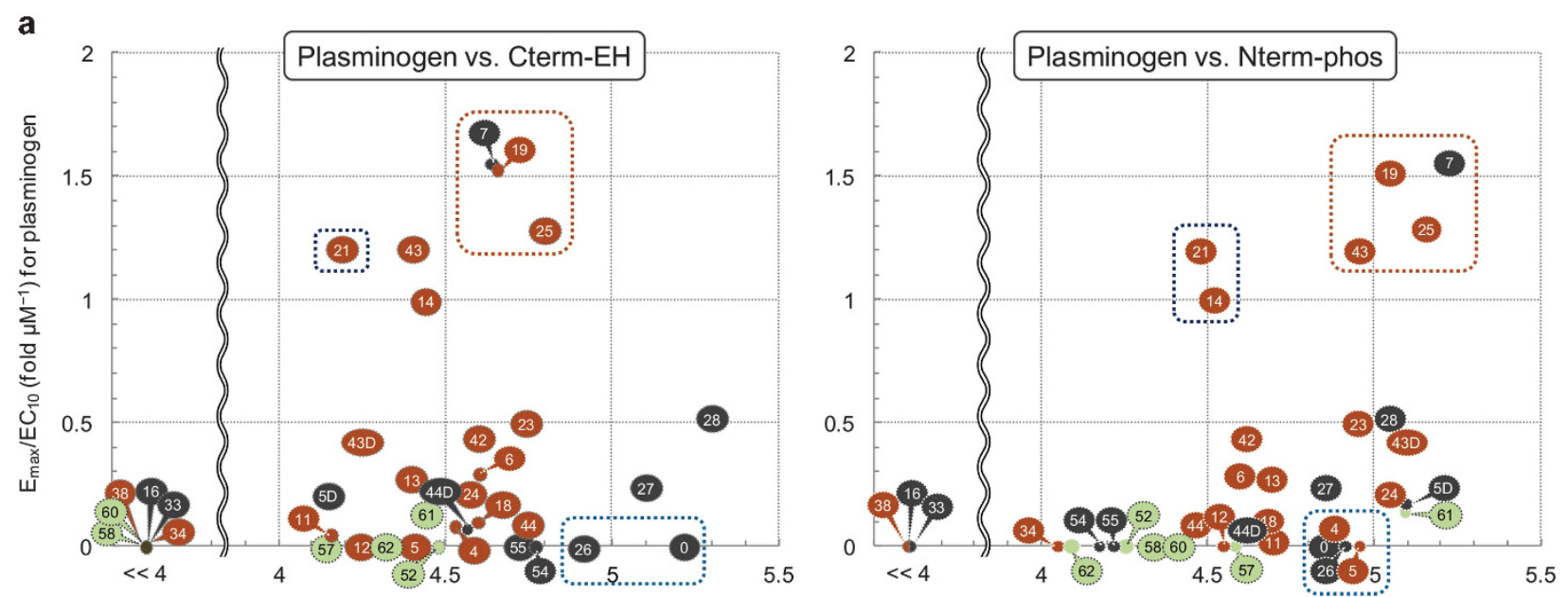

b

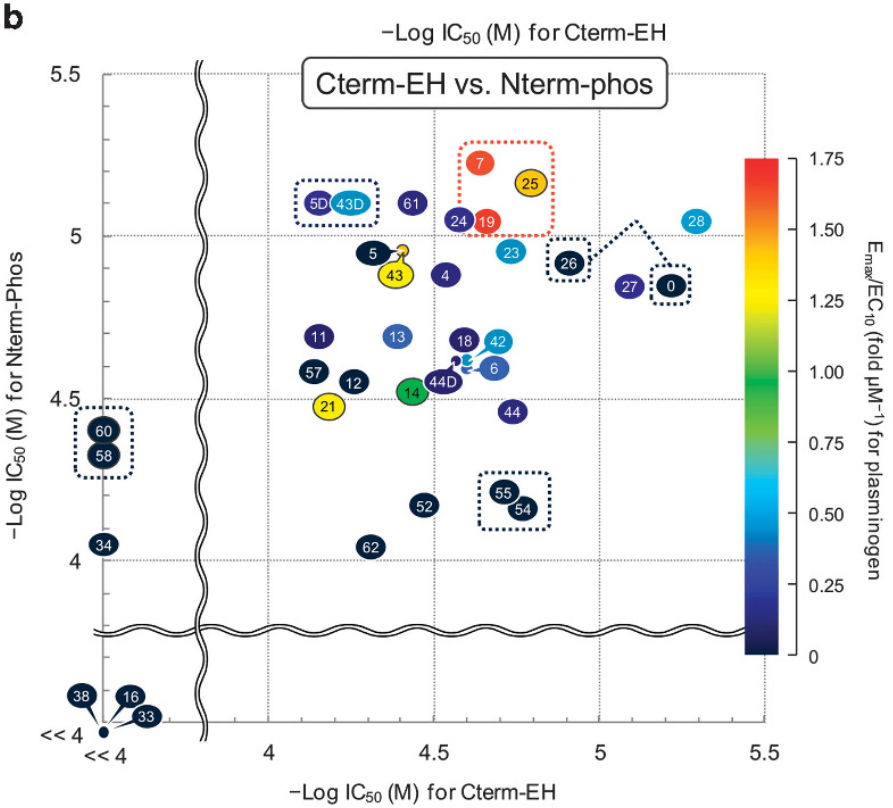

C

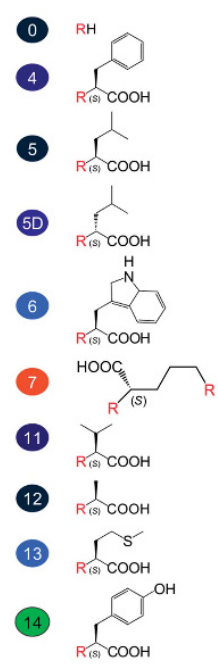

- $\log \mathrm{IC}_{50}(\mathrm{M})$ for Nterm-phos

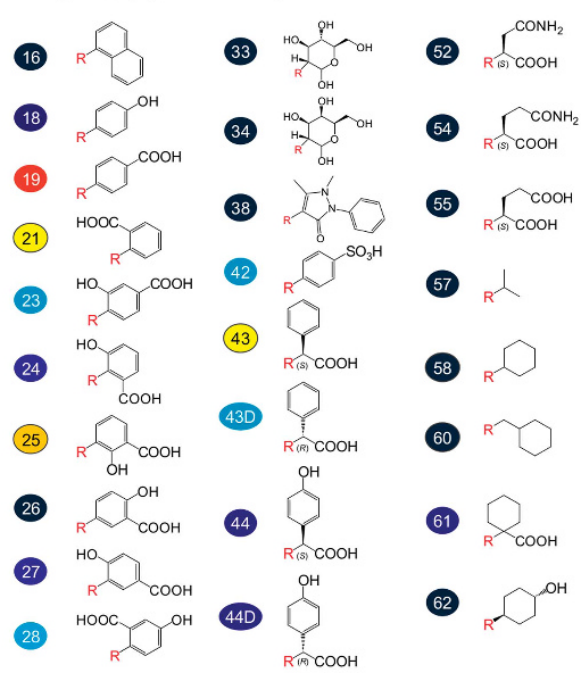

Figure 2 Structure-activity relationships of SMTP congeners with respect to plasminogen modulation and inhibitions of Cterm-EH and Nterm-phos of soluble epoxide hydrolase $(\mathrm{sEH})$. (a) Structure-activity relationships with respect to plasminogen modulation and Cterm-EH (left panel) and to plasminogen modulation and Nterm-phos (right panel). The numbers in the circles represent SMTP numbers. The colors of the circles represent congeners as follow: pale olive, new ones; dark blue, previously characterized ones; dark red, newly characterized ones. Characteristic congeners are encircled in a dashed line. (b) Structure-activity relationships with respect to sEH inhibition in combination with plasminogen modulation. Cterm-EH inhibition is plotted against Ntermphos inhibition with numbered symbols as in a. The colors indicate the potency of plasminogen modulation activity as shown in the right bar. Characteristic congeners are encircled in a dashed line. (c) Structures of the $N$-linked side chain of the congeners tested are shown. Red 'R' represents the core structure shown in Figure 1.

activity in treating thrombotic and embolic stroke. ${ }^{17-21}$ Therefore, a congener with the three activities may be useful for developing a drug for thromboembolic diseases. A congener without plasminogen modulation but inhibitory to both or either of the activities of $\mathrm{sEH}$ may be of use in other indications described above.

\section{ACKNOWLEDGEMENTS}

Human plasma for plasminogen isolation was provided by the Japanese Red Cross Society, Tachikawa. This work was supported in part by grants from the Japan Science and Technology Agency (grant number AS2316911G to KH), the Institute for Fermentation, Osaka, Japan (to KH) and the Japan Society for the Promotion of Science (grant number 26750362 to ES).
1 Hasumi, K., Yamamichi, S. \& Harada, T. Small molecule modulators of the zymogen activation in the fibrinolytic and coagulation systems. FEBS J. 277, 3675-3687 (2010).

2 Shinohara, C., Hasumi, K., Hatsumi, W. \& Endo, A. Staplabin, a novel fungal triprenyl phenol which stimulates the binding of plasminogen to fibrin and U937 cells. J. Antibiot. 49, 961-966 (1996).

3 Kohyama, T., Hasumi, K., Hamanaka, A. \& Endo, A. SMTP-1 and -2, novel analogs of staplabin produced by Stachybotrys microspora IF030018. J. Antibiot. 50, 172-174 (1997).

4 Takayasu, R., Hasumi, K., Shinohara, C. \& Endo, A. Enhancement of fibrin binding and activation of plasminogen by staplabin through induction of a conformational change in plasminogen. FEBS Lett. 418, 58-62 (1997).

5 Hasumi, K. et al. Isolation of SMTP-3, $-4,-5$ and -6 , novel analogs of staplabin, and their effects on plasminogen activation and fibrinolysis. J. Antibiot. 51, 1059-1068 (1998)

$6 \mathrm{Hu}$, W., Ohyama, S. \& Hasumi, K. Activation of fibrinolysis by SMTP-7 and -8 , novel staplabin analogs with a pseudosymmetric structure. J. Antibiot. 53, 241-247 (2000). 
7 Hu, W., Kitano, Y. \& Hasumi, K. SMTP-4D, -5D, -6D, -7D and -8D, a new series of the non-lysine-analog plasminogen modulators with a D-amino acid moiety. J. Antibiot 56, 832-837 (2003).

8 Hasumi, K., Hasegawa, K. \& Kitano, Y. Isolation and absolute configuration of SMTP-O, a simplest congener of the SMTP family nonlysine-analog plasminogen modulators. $J$ Antibiot. 60, 463-468 (2007).

9 Ohyama, S., Harada, T., Chikanishi, T., Miura, Y. \& Hasumi, K. Nonlysine-analog plasminogen modulators promote autocatalytic generation of plasmin(ogen) fragments with angiostatin-like activity. Eur. J. Biochem. 271, 809-820 (2004).

10 Hasegawa, K. et al. Structure-activity relationships of 11 new congeners of the SMTP plasminogen modulator. J. Antibiot. 63, 589-593 (2010).

11 Koide, H., Narasaki, R., Hasegawa, K., Nishimura, N. \& Hasumi, K. A new series of the SMTP plasminogen modulator with a phenylglycine-based side-chain. J. Antibiot. 65, 91-93 (2012).

12 Koide, H., Hasegawa, K., Narasaki, R., Nishimura, N. \& Hasumi, K. A new series of the SMTP plasminogen modulators with a phenylamine-based side-chain. J. Antibiot. 65 361-367 (2012)

13 Nishimura, Y. et al. Pre-SMTP, a key precursor for the biosynthesis of the SMTP plasminogen modulators. J. Antibiot. 65, 483-485 (2012)

$14 \mathrm{Hu}$, W., Narasaki, R., Nishimura, N. \& Hasumi, K. SMTP (Stachybotrys microspora triprenyl phenol) enhances clot clearance in a pulmonary embolism model in rats. Thromb. J. 10, 2 (2012)

15 Koyanagi, K., Narasaki, R., Yamamichi, S., Suzuki, E. \& Hasumi, K. Mechanism of the action of SMTP-7, a novel small-molecule modulator of plasminogen activation. Blood Coagul. Fibrinolysis 25, 316-321 (2014).

16 Miyazaki, T. et al. Distinct effects of tissue-type plasminogen activator and SMTP-7 on cerebrovascular inflammation following thrombolytic reperfusion. Stroke 42, 1097-1104 (2011)

17 Shibata, K., Hashimoto, T., Nobe, K., Hasumi, K. \& Honda, K. A novel finding of a lowmolecular-weight compound, SMTP-7, having thrombolytic and anti-inflammatory effects in cerebral infarction of mice. Naunyn Schmiedebergs Arch. Pharmacol. 382, 245-253 (2010)

18 Hashimoto, T., Shibata, K., Nobe, K., Hasumi, K. \& Honda, K. A novel embolic model of cerebral infarction and evaluation of SMTP-7, a novel fungal triprenyl phenol metabolite. J. Pharmacol. Sci. 114, 41-49 (2010).

19 Akamatsu, Y. et al. Stachybotrys microspora triprenyl phenol-7, a novel fibrinolytic agent, suppresses superoxide production, matrix metalloproteinase- 9 expression, and thereby attenuates ischemia/reperfusion injury in rat brain. Neurosci. Lett. 503, 110-114 (2011)

20 Sawada, H. et al. SMTP-7, a novel small-molecule thrombolytic for ischemic stroke: a study in rodents and primates. J. Cereb. Blood Flow Metab. 34, 235-241 (2014)
21 Ito, A. et al. SMTP-7, a new thrombolytic agent, decreases hemorrhagic transformation after transient middle cerebral artery occlusion under warfarin anticoagulation in mice. Brain Res. 1578, 38-48 (2014)

22 Shibata, K., Hashimoto, T., Nobe, K., Hasumi, K. \& Honda, K. Neuroprotective mechanisms of SMTP-7 in cerebral infarction model in mice. Naunyn Schmiedebergs Arch. Pharmacol. 384, 103-108 (2011).

23 Hashimoto, T., Shibata, K., Ohata, H., Hasumi, K. \& Honda, K. Altered gene expression in an embolic stroke model after thrombolysis with tissue plasminogen activator and Stachybotrys microspora triprenyl phenol-7. J. Pharmacol. Sci. 125, 99-106 (2014).

24 Donnan, G. A., Fisher, M., Macleod, M. \& Davis, S. M. Stroke. Lancet 371, 1612-1623 (2008).

25 Donnan, G. A. et al. How to make better use of thrombolytic therapy in acute ischemic stroke. Nat. Rev. Neurol. 7, 400-409 (2011)

26 Matsumoto, N., Suzuki, E., Ishikawa, M., Shirafuji, T. \& Hasumi, K. Soluble epoxide hydrolase as an anti-inflammatory target of the thrombolytic stroke drug SMTP-7. J. Biol. Chem. 289, 35826-35838 (2014).

27 Newman, J. W., Morisseau, C. \& Hammock, B. D. Epoxide hydrolases: their roles and interactions with lipid metabolism. Prog. Lipid Res. 44, 1-51 (2005).

28 Gomez, G. A., Morisseau, C., Hammock, B. D. \& Christianson, D. W. Structure of human epoxide hydrolase reveals mechanistic inferences on bifunctional catalysis in epoxide and phosphate ester hydrolysis. Biochemistry 43, 4716-4723 (2004).

29 Michaelis, U. R. \& Fleming, I. From endothelium-derived hyperpolarizing factor (EDHF) to angiogenesis: epoxyeicosatrienoic acids (EETs) and cell signaling. Pharmacol. Ther. $111,584-595$ (2006)

30 Spector, A. A. \& Norris, A. W. Action of epoxyeicosatrienoic acids on cellular function. Am. J. Physiol. Cell Physiol. 292, C996-1012 (2007).

$31 \mathrm{lliff}$, J. J. et al. Epoxyeicosanoid signaling in CNS function and disease. Prostaglandins Other Lipid Mediat. 91, 68-84 (2010)

32 Inceoglu, B. et al. Soluble epoxide hydrolase and epoxyeicosatrienoic acids modulate two distinct analgesic pathways. Proc. Natl Acad. Sci. USA 105, 18901-18906 (2008)

33 Tran, K. L. et al. Lipid sulfates and sulfonates are allosteric competitive inhibitors of the $\mathrm{N}$-terminal phosphatase activity of the mammalian soluble epoxide hydrolase. Biochemistry 44, 12179-12187 (2005)

34 Morisseau, C. et al. Role of soluble epoxide hydrolase phosphatase activity in the metabolism of lysophosphatidic acids. Biochem. Biophys. Res. Commun. 419, 796-800 (2012)

35 Oguro, A. \& Imaoka, S. Lysophosphatidic acids are new substrates for the phosphatase domain of soluble epoxide hydrolase. J. Lipid Res. 53, 505-512 (2012).

36 Harris, T. R. \& Hammock, B. D. Soluble epoxide hydrolase: gene structure, expression and deletion. Gene 526, 61-74 (2013).

Supplementary Information accompanies the paper on The Journal of Antibiotics website (http://www.nature.com/ja) 